\title{
Carole Bourne-Taylor
}

\section{Imaginaire identitaire/identité imaginaire dans Meursault, contre-enquête de Kamel Dooud: question(s) de démêlés...}

THE IMAGINARY OF IDENTITY/IMAGINARY IDENTITY IN KAMEL DaOUd'S MEURSAULT CONTRE-ENQUÊTE: ISSUES AND ENTANGLEMENTS

Abstract: Identity is approached from the perspective of the genre of literary counterinvestigation, in the wake of Laurent Demanze's study Un nouvel âge de l'enquête. In post-colonial Algeria, the motif of spectrality is bound up with an imaginary of grief and impossible mourning, typified by Kamel Daoud's Meursault, contre-enquête. In this context, identity is synonymous with alienation. Like his predecessors Camus and Assia Djebar, Daoud is conducting his own quest for freedom, promoting the relational and ethical value of the imaginary as a universal network of images that counter the narrow enclosure of nationalist and fundamentalist discourse in a fundamentally dynamic encounter with the world and the other.

Keywords:Kamel Daoud; Albert Camus; Identity; Algeria; Post-colonialism; Counterinvestigation; Grief; Ethical Imaginary; Myth.

\section{CAROLE BOURNE-TAYLOR}

Brasenose College, Oxford University

carole.bourne-taylor@bnc.ox.ac.uk

DOI: 10.24193/cechinox.2021.41.18 ue la question de l'identité se pose avec
acuité dans l'enquête littéraire, a fortiori la contre-enquête, n'est guère surprenant. Le roman de Kamel Daoud, Meursault, contre-enquête, publié en 2013 en Algérie, puis en 2014 en France ${ }^{1}$, brillante trace de la diffraction de l'incontournable héritage camusien, en est une preuve patente. «Question(s) de démêlés » car il s'agit, pour Haroun, son narrateur endeuillé, d'éclaircir une affaire compliquée, voire l'un des grands mystères ${ }^{2}$ de la littérature de langue française, si tant est que cela soit possible ; "démêlés " dans le sens de débat et dans son sens juridique, évidemment, puisque la contre-enquête, toujours contestataire, consiste à réclamer justice ${ }^{3}$, en l'occurrence à demander réparation pour l'Arabe abattu dans L'Étranger de Camus. Ce meurtre est un "détour " ${ }^{4}$ élaboré par la fiction pour effectuer le deuil impossible ou " oblique » dont le roman de Camus serait le modèle. ${ }^{5}$ Il s'agit d'une autre exploration - ou plutôt d'une exploration autre - de l'imaginaire de la douleur, dans l'entre-deux de la malédiction et de la contradiction : là résident les enjeux identitaires de la contre-enquête. 
Toute lecture, toute écriture, toute réécriture s'élabore sous le signe de la hantise, corollaire de l'héritage - le motif de la spectralité est d'ailleurs amplement exploité dans le texte daoudien. Cette conscience critique d'un héritage qui participe d'une démarche phénoménologique d'entrelacement de destinées (individuelle et collective, nationale et transnationale, présente et passée), a trouvé un écho dans de belles fictions identitaires sous-tendues par une dialectique de déconstruction/reconstruction, parmi lesquelles Le dernier été d'un jeune homme de Salim Bachi et Aujourd'hui Meursault est mort de Salah Guemriche. Ladmiration pour Camus s'y accompagne d'un souci d'honorer leur frère mort. Autant de variations sur la même énigme. C'est dans le contexte d'une revendication identitaire postcoloniale, intertextuelle et interculturelle, qu'il convient d'aborder Meursault, contre-enquête : cette entreprise de réhabilitation passe par la réécriture, qui, à son tour, passe par la nomination. Frère de l'Arabe abattu par Meursault dans L'Étranger en 1942, Haroun, un Algérien parvenu à l'heure des bilans, raconte l'histoire de son frère, "l'histoire d'un crime ", « absurde $»^{6}$, à un doctorant de passage, soixante-dix ans après cette mort, dans un bar d'Oran. Ce déséquilibre entre un Meursault $^{7}$ si célèbre et la victime anonyme, l'Arabe mort sans sépulture, "dans l'insignifiance, tel un vulgaire figurant $»^{8}$, est ressenti comme une injustice. "Il n'y a pas trace de notre deuil ${ }^{9}$ : c'est là que le bât blesse. Cruelle déception et amertume de Haroun qui croyait retrouver, en lisant L'Autre, toute une phénoménologie qui aurait pu lui rendre « les derniers mots de mon frère, la description de son souffle, ses répliques face à l'assassin, ses traces et son visage $» .{ }^{10}$ Or rien. Le plus urgent, c'est de nommer Moussa (appellation arabe de Moïse) pour remédier à cette aberration intolérable de l'anonymat. Il convient bien de parler de sidération ${ }^{11}$, donc, face à ce manque de considération, tandis que le mot "Arabe », relégué à sa fonction générique métonymique, est soumis à la répétition vide, procédé de l'absurde. Cercle vicieux car pour conjurer cette béance, Haroun lui oppose la répétition incantatoire du nom : «Moussa, Moussa, Moussa... ». ${ }^{12}$ Le but de la contre-enquête est la restitution de l'identité occultée, oblitérée du disparu, ainsi que celle du survivant qui doit se forger une identité contre, une identité qui est en fait étrangeté (de manière poignante, à soi-même dans son pays natal). Étranger, Haroun devient aussi le double de l'Autre ( L'étranger » de Camus ${ }^{13}$ ), identité qu'il revendique, comme il revendique d'ailleurs l'absurde ${ }^{14}$ allant jusqu'à le pousser $a d$ absurdum, comme en témoigne l'attribution du nom de Moussa à tous ${ }^{15}$, pluralisation qui est un geste désespéré et ironique. Après tout, dans L'Autre, "Moussa est un Arabe que l'on peut remplacer par mille autres de son espèce $"{ }^{16}$ Un drôle de jeu de miroirs se met en place, révélateur d'une crise identitaire - spectralité et spécularité étant inextricables. La fortune de la contre-enquête est liée à un contexte tendu et tourmenté. Le texte de Daoud présente une énigme non résolue sur l'arrière-fond du drame colonial et de ses ramifications post-coloniales. La reconstitution mémorielle rouvre une plaie de l'Histoire. Nous nous aventurons dans l'imaginaire (terme récurrent) d'un narrateur-enquêteur torturé par ses traumatismes et aiguillonné par ses fantasmes; nous explorons ses autoreprésentations sans toutefois perdre 
de vue la dimension collective des enjeux identitaires. Bien que le roman mette en jeu une confrontation à l'Autre colonial, il n'est pas question de le réduire à une polémique postcoloniale même s'il met en œuvre un imaginaire postcolonial ; ce serait un appauvrissement qui trahirait la logique d'ouverture emblématique de Daoud, plus soucieux, comme Camus, de poser des questions que de fournir des réponses.

Cette contre-enquête forcenée consiste en une réécriture qui vise à combler les blancs de l'écriture blanche de L'Étranger de Camus, un " mensonge cousu de fil blanc " puisque l'Arabe qui aurait dû en être un protagoniste majeur, «n'y est même pas tué » puisqu'il « n'a ni nom, ni visage, ni paroles. $"{ }^{17}$ Peut-être conviendrait-il de convoquer la métaphore $\mathrm{du}$ «blanc de l'Algérie » pour reprendre le titre du récit d'Assia Djebar, publié en 1995 en hommage à « ces chers disparus ${ }^{18}$, parmi lesquels des victimes de la " décennie noire » de la terreur islamiste. Les textes de Daoud et de Djebar sont des chants de deuil et des récits de fantômes jouant sur les registres dramaturgiques et poétiques et sur le chromatisme ambigu du blanc - le blanc irréel de l'effacement, métonymie et hypostase du deuil dans Alger la Blanche. Dans le roman de Daoud, la mort, la mer ${ }^{19}$ et la mère (il y a plus qu'une harmonie phonique entre les deux, de même que le nom Meursault se prête à des jeux sur les signifiants mer, mort et soleil), le soleil aveuglant (ce même soleil fatal dont la mer se fait le complice du meurtre de Meursault), le silence et l'inachèvement (comme le deuil interminable) forment un écheveau métaphorique inextricable. Le blanc (comme le soleil, comme la mer) est à double tranchant puisque, ultimement, il représente aussi la page blanche où l'imaginaire peut se déployer tous azimuts.
La contre-enquête prend son élan dans l'ignorance douloureuse, dans le nondit, l'impensé et l'impensable, qui affleure sous forme de traces (le terme est un leitmotiv), d'indices, de lambeaux, de cicatrices qui configurent le réseau imaginal d'une mémoire mutilée et troublée en même temps qu'ils constituent cette identité à jamais déchirée. Il s'agit de désenfouir ce qui plombe l'existence : le paradoxe d'une absence encombrante, d'un vide trop plein - comme ce soleil éblouissant ou ce silence nocturne qui laisse entendre " le vacarme de ses définitions multiples $»{ }^{20}$ Les motifs éminemment symboliques et récurrents de l'abîme ${ }^{21}$ et du vide figurent l'engouffrement identitaire.

Louvrage de Laurent Demanze, Un nouvel âge de l'enquête, montre que ce regain d'intérêt pour l'enquête, a fortiori la contre-enquête, est symptomatique d'une époque en proie à des questionnements identitaires lancinants. Dans le texte qui nous intéresse, ceux-ci se nouent autour d'un imaginaire du deuil, creuset d'une identité complexe, voire impossible à démêler. Ce deuil pèse comme une malédiction sur Haroun qui passe son existence à débattre et à se débattre entre ces deux pôles identitaires que sont la malédiction et la contradiction. D'ailleurs, c'est l'interlocution qui permet ce va-et-vient, qui permet d'imaginer ou de réimaginer l'identité placée sous le signe de la malédiction, se forgeant, tant bien que mal, une délivrance dans la contradiction, c'est-à-dire par les signes. La prééminence du narrateur est trompeuse, qui annonce la disqualification de la posture empêtrée dans le ressassement ${ }^{22}$ de $"$ cette histoire ${ }^{23}$ proférée par une voix apparemment incapable de s'inscrire en tant que sujet autonome et 
souverain, la voix d'une identité vampirisée ou parasitée. Le référent frappé d'anonymat pendant des décennies a tout loisir de se spectraliser; ne bénéficiant d'aucune immunité, Haroun, quant à lui, est happé par l'identité fantomatique de Moussa. Concept psychanalytique qui révèle la persistance du traumatisme, le fantôme est "l'invention des vivants, le résultat de la lacune qu'a créé en nous l'occultation d'une partie de la vie d'un objet aimé. $»^{24}$ L'« imaginaire spectral $»^{25}$ qui s'est répandu dans la littérature de la dépossession ou de la disparition depuis les années quatrevingt, marquées par le retour de l'histoire et du sujet, ${ }^{26}$ imprègne la littérature postcoloniale. L'altérité spectrale du narrateur doit se comprendre en regard du syndrome de la disparition qui agit comme une contamination, ou plutôt comme une malédiction (motif obsessionnel): être arabe constitue une généricité spectrale. L'identité individuelle est toujours prise dans des questions de filiation, d'un emboîtement indivis : «l'Arabe, $[\ldots]$ sa famille, $[\ldots]$ son peuple ${ }^{27}$ Orphelin qui porte sa survivance comme un fardeau, Haroun est le produit d'une piètre généalogie ${ }^{28}$, issu d'un père volatilisé « depuis des siècles » $^{29}:$ «moi, Haroun, frère de Moussa, fils du père disparu $»{ }^{30}$ Outre l'autophobie patente du personnage, tout contribue à l'intériorisation d'une image de soi comme être dépossédé dans un pays ravagé. De surcroît, cette condition d'orphelin est clivée puisque Haroun est tiraillé entre deux filiations également lacunaires et toxiques : «Meursault et sa mère d'une part ; M'ma et Moussa de l'autre $»{ }^{31}$ Véritablement " maudit $»^{32}$, Haroun apparaît comme la victime d'une évanescence identitaire, d'une aliénation compliquées par l'altérité tératologique d'une mère "féroce ${ }^{33}$ et "folle $»^{34}$ qui l'embarque dans ses enquêtes, " rite contre la douleur ${ }^{35} \mathrm{Si}$ la mère est « encore vivante ${ }^{36}$, c'est son deuil «éternel $\aleph^{37}$ qui paradoxalement la maintient en vie, une vie spectrale et sépulcrale: une mère morte vivante, une " mère monstrueuse ${ }^{38}$ et incestueuse, qui contamine Haroun de sa propre déshérence et dont les «monstrueux flots d'histoires inventées ${ }^{39}$ spectralisent les vivants et prêtent vie aux spectres. Il faut dire que l'étrangeté constitue le grand dénominateur commun, un drôle d'entrelacs phénoménologique. Le martyre de cette mère qui « aurait pu avoir une pension de veuve de martyr $~^{40}$, surnommée " l'armala " (la veuve $)^{41}$, est la métonymie d'une Algérie non-guérie. Quant à leur bicoque, Haroun la compare à une tombe ou à un "ventre malade ». ${ }^{42}$ À cet égard, il convient de souligner le symbole prégnant du labyrinthe ${ }^{43}$, lieu d'une violence archaïque, d'une expérience tragique de l'espace qui fait partie intégrante de la constitution de l'identité ; ajoutons-y la vision d'un Oran grouillant de rats, tout droit sorti de La Peste. ${ }^{44} \mathrm{Le}$ mythe essaime, comme en témoigne la structure labyrinthique de la confession dont on perd le fil et qui en dit long sur cette quête identitaire impossible. Deuil d'autant plus interminable que la dignité de cette famille a été bafouée. Insoutenable légèreté de cette identité dont l'inanité contraste avec le fardeau du deuil : «Arabe, je ne me suis jamais senti arabe, tu sais. C'est comme la négritude qui n'existe que par le regard du Blanc. Dans le quartier, dans notre monde, on était musulman, on avait un prénom, un visage et des habitudes ». ${ }^{45}$ Face aux « étrangers », aux roumis (dans le lexique musulman), les Algériens étaient condamnés à «flott[er] dans l'angle 
mort du paysage ${ }^{46}$, si bien que la tentative de se hisser à l'identité s'avère bien pénible, même à l'ère postcoloniale, comme le suggère cette autodéfinition bien dérisoire : " marginal " ${ }^{47}$, " célibataire, sombre et mutique » et aussi " lâche ${ }^{48}$ aux yeux des Frères. Drôle de vie par procuration pour Haroun dont la mère exige qu'il endosse l'identité de Moussa. Il y a un va-et-vient constant entre son identité et celle du mort, interchangeables dans leur inconsistance spectrale, dans l'entre-deux d'un remâchage mélancolique ponctué des leitmotivs « fantôme » et « ombre ». Haroun est conscient que l'histoire de Moussa, c'est son histoire, l'histoire d'une désubjectivation : ni plus ni moins qu' « $[\mathrm{u}]$ ne autre histoire d'Arabe $"{ }^{49}$ Enfant maladif, Haroun doit apprendre à refouler ses désirs au point de nier son corps. La récurrence du fantasme de dévoration et de manducation (dont l'exemple parlant du besoin de dérober le sucre pour prouver son existence à cette mère revêch $\mathrm{e}^{50}$ ) appelle une interprétation psychanalytique. La mère castratrice proférant ses malédictions ne se contente pas d'accabler Haroun du fardeau d'un deuil impossible, elle le pousse aussi au " contrecrime » vengeur, en 1962 , ce qui renforce son sentiment d'être "condamné " ${ }^{51}$ : un pas supplémentaire dans la malédiction; ironiquement, absurdement, sa vengeance lui sera d'ailleurs déniée. Le dédoublement se radicalise, Haroun se retrouve face à luimême et à son meurtre pour un jugement impitoyable. Pour sa défense imaginaire, il dit s'être « enterré vivant » afin que sa mère " vive d'espoir " ${ }^{52}$ : ultime sacrifice. "Au fond, j'ai vécu plus tragiquement que ton héros $»^{53}$, lance-t-il, sur un ton provocateur, à son interlocuteur. Ce sujet pris dans une histoire toujours plus ou moins noire (de la tourmente coloniale à la dysphorie, voire dystopie, post-coloniale) a du mal à se sentir exister.

Daoud évoque une " mémoire dévorante ", " l'entrave paralysante de la mémoire et le trauma d'une guerre dont le souvenir est devenu une identité en soi $»{ }^{54}$ Le motif du deuil est indissociable des traumatismes de l'Histoire qui se perpétuent, phagocytant l'identité individuelle dont il est la composante constitutive. Moussa aurait pu devenir un martyr, et Haroun un héros, mais celui-ci devient de plus en plus fantomatique, plus encore que ne l'est son frère. Ses démêles avec le Front de Libération Nationale pour avoir tué le Français un jour trop tard montrent bien que l'identité, chez Daoud, comme chez Camus, n'échappe pas à l'absurdité. La libération s'énonce sur le mode du contrefactuel, c'est-à-dire comme un possible larvé, fantasmé mais non-accompli.

L'imaginaire déborde du format de la contre-enquête, aussi fructueux que soit ce schéma. Haroun lui-même en prend conscience lorsqu'il formule le vœu d'écrire un livre : "il ne s'agirait pas d'une contre-enquête sur le cas de ton Meursault, mais d'autre chose, de plus intime ", avant de lancer cette boutade : « [u]n grand traité de la digestion $» .{ }^{55}$ Serions-nous donc dans l'antiphrase ? Non, on est tout simplement dans une littérature qui fait fi des catégories, cultive l'ambiguïté et le paradoxe, brouille les pistes et emmêle plus qu'elle ne démêle. La contre-vérité est l'envers de la vérité, celle-ci étant l'objet d'une quête forcément inachevable. On connaît la fortune du paradigme du « à ce qui n'en finit pas » pour dire l'infini du deuil. ${ }^{56} \mathrm{Il}$ y a bien « contre-enquête » mais elle ne peut aboutir, sauf en tant que transfert imaginaire: 
" Faire vivre Moussa après avoir été mort, à sa place. " ${ }^{57} \mathrm{La}$ " contre-enquête " relève donc d'un régime de l'exploration du possible: «Cela a mené à une sorte de livre étrange - que j'aurais peut-être dû écrire d'ailleurs, si j'avais eu le don de ton héros. " ${ }^{58}$ Haroun est lucide : il s'est fait vampiriser par une mère dont la voracité vengeresse est semblable au tonneau des Danaïdes. La vie fantomatique de Moussa et la mort symbolique de Haroun sont les versants d'une même fatalité. Voué à « un strict devoir de réincarnation ", Haroun vit " une enfance de revenant $~^{59}$ dont l'éveil sensuel se fait au cimetière ${ }^{60}$ : il finit par devenir " la trace du mort ${ }^{61}$, lui dont le seul devoir et destin est de " faire vivre Moussa après avoir été mort, à sa place $»{ }^{62}$ Il construit cette cosmogonie fabuleuse dans laquelle il s'abîme dans tous les sens du terme : dans sa douleur, dans son deuil, dans la reconstruction imaginaire, mais aussi de manière plus dramatique et tragique même si ce sont désormais les Français qui sont devenus des "spectres ». ${ }^{63}$ Tout Français est d'emblée perçu comme un "vétéran " dans cette Algérie à jamais hantée par une France promue au rang d'" Imago mundi indépassable ". ${ }^{64}$ Nous sommes dans l'aporie gordienne.

Démêler le fil sémantique du terme « démêlés » est loin d'être une entreprise aisée : il évoque une contestation qui devrait donner lieu à des éclaircissements, ainsi qu'une source d'incessants débats. Dans ce genre de démarche archéologique, les catégories sont emmêlées (fiction et autobiographie, mémoire personnelle et mémoire collective, passé et présent), et le retour passe par le détour identitaire. Le retour à L'Étranger figure le détour nécessaire par « l'étranger» en tant que principe dynamique, heuristique qui ouvre l'identité à l'altérité. Tout un imaginaire inter- et trans-relationnel (qui englobe la filiation et l'intertextualité, laquelle fait rimer identité et ubiquité) informe le roman. Traquer les enjeux identitaires du roman de Daoud nous amène à réaffirmer le pouvoir relationnel, empathique et éthique de la littérature.

La construction identitaire transite souvent par la lecture. Dans l'Algérie des années 1990, Daoud renie l'islamisme pour la littérature qui propose une autre manière de construire du sens, un autre usage du mythe, une démythification au sens de démystification des mythes, une réactualisation, et, enfin, une libération qui a des répercussions identitaires ; en effet, pendant la décennie noire, le Front Islamiste du Salut (FIS) s'était assuré le monopole de l'identité musulmane décrétée seule et unique identité légitime, niant ainsi la diversité ethnoculturelle (berbère, turque, romaine et arabe) : retour à un nouvel essentialisme, à un nouvel enfermement après la domination coloniale. La conversion daoudienne de la religion à la littérature (c'est-à-dire d'un usage délirant à un usage délivrant du mythe) retrace la métamorphose d'un goût pour le mysticisme car Daoud croit aux mythes.

C'est la découverte de l'absurde, tel qu'il fut formulé par Camus, qui détermina le changement de cap. Dans ce texte palimpsestique, l'intertextualité est l'un des avatars de l'altérite polymorphe, métissée (telle est la fonction des arabismes). Daoud subtilise les mythes, les revivifie, les délocalise afin de réimaginer l'identité. C'est ainsi qu'il convoque une gamme de textes sacrés, récits des origines, mythes (dont L'Iliade) : tous s'y côtoient, incarnés par Aaron, 
Moïse, Myriam, Abel et Cain, mais aussi Robinson Crusoé et sa logique coloniale. Toutes ces sources participent d'un brassage onirique atemporel. D'ailleurs la tradition mythique de la robinsonnade (et ses variations chez Tournier, Chamoiseau et Coetzee, par exemple) est issue d'un conte arabe du XII ${ }^{e}$ siècle, lui-même occulté. C'est par l'altérité (au sens le plus élastique du terme) que l'on construit son identité ; la rivalité au sein de la fraternité, écho de la Bible et du Coran, est complexifiée par le brouillage entre fraternité et étrangeté. La référence à Orphée ${ }^{65}$, force médiatrice, symbolise la fluidité entre le règne des vivants et celui des morts, en même temps quelle reprend la thématique du veuvage inconsolable et affirme la réconciliation entre Apollon et Dionysos et, par-dessus tout, l'inachèvement propre à cette mythographie fondamentalement complexe, à la fois synthétique et contradictoire.

La conversion de Daoud est une pulsion de vie, un hymne à la vie - impossible de ne pas songer à Camus. Justement, " piégé entre la mère et la mort " ${ }^{66}$, "partagé », comme il le dit, " entre ces pôles de fascination » que sont « [1]a mère, la mort, l'amour $\aleph^{67}$ qui glissent au gré des signifiants, Haroun sait reconnaître l'amour (qui est aussi l'amour de la vie) incarné par Meriem ; c'est une échappatoire, un antidote à l'amour de la mère (qui a partie liée avec la mort). Faisant irruption dans la vie de Haroun comme un rayon de soleil trop ardent, Meriem enquête sur "l'Arabe " pour les besoins d'une thèse sur le roman de Meursault (L'Autre). La première à prononcer le nom de Moussa Ould el-Assasse ${ }^{68}$, Meriem le ressuscite en un speech-act cathartique et ironique qui, comme un précipité chimique, ne fait que révéler la fatalité qui pèse sur Haroun. Principe vital, Meriem est décrite comme un « soleil candide et incandescent " $"$ : 1 la solarité se trouve atténuée par la grâce de l'oxymore. Meriem aurait pu sauver Haroun puisquelle est la seule qui ait pu " [le] ramener à la vie $»^{70}$ dans leuphorie de la post-indépendance. Incarnation sensorielle paroxystique, synesthésique ${ }^{71}$, elle est l'antithèse de la réalité mortifère dans laquelle se débat Haroun ; elle est « l'incandescence, le désir, la rêverie, l'attente, l'affolement des sens $»^{72}$ : c'en est trop pour Haroun qui dit aimer et hair Meriem dans un passage qui met en œuvre un renversement ironique de L'Étranger puisque elle a perturbé son équilibre (un leitmotiv). La passion paradoxale de Haroun pour Meriem relève de la fascination, qui court-circuite la conscience par son intense réceptivité. Le motif obsédant de l'incandescence (qui symbolise le scandale de lépanouissement de la chair au soleil, une phénoménologie scandaleuse du corps et de la sensualité) transforme Meriem en une hallucination. L'imaginaire de la perte engloutit jusqu'à la moindre promesse de bonheur. Il semble que tout soit voué à la perte, qui serait l'alpha et l'oméga.

Radieuse, Meriem représente un idéal inaccessible, la liberté qui ne peut être que rêvée ; ainsi bascule-t-elle ironiquement dans le régime spectral du « ce qui aurait pu être ». Elle ne surgit que pour aiguiser ou exacerber (plutôt qu'exorciser) le manque : dispositif narratif ingénieux qui instrumentalise Meriem, belle et rebelle, emblème d'une féminité libérée ${ }^{73}:$ trans- $^{-}$ gression suprême, suprême ironie de faire d'elle une créature si magnétique, mais énigmatique. Haroun ne vit véritablement que dans les moments qu'il passe 
avec Meriem, dans ces intermittences du cœur ; un amour qui contraste avec les modalités dégénérescentes du corps (mort, évanescence spectrale, maladie, sénescence aigrie). Haroun est écartelé entre le monstre nyctomorphe (sa mère) et le sensualisme lumineux et purificateur d'une Meriem promesse de vie, une vie, qui (on l'imagine) sera niée par le sexisme. Si sa beauté harponne Haroun, cette possibilité de vie s'avère être une chimère. Le motif insistant de l'incandescence la désigne à la fois comme un surplus d'identité et comme un mirage. Providentielle ${ }^{74}$ seulement au conditionnel passé, emblème d'un paradoxe indépassable, Meriem ne peut exorciser Haroun qui est prisonnier de sa spectralité ${ }^{75}$. L'ironie ultime veut que le coup de foudre soit vécu comme une malédiction. ${ }^{76}$ Haroun oscille entre l'épreuve de la perte et du manque (un régime d'outre-tombe) d'une part et l'ivresse de la transgression (l'hédonisme subversif représenté par l'alcool et l'amour) d'autre part, qui est, en fin de compte, une autre perte. Il incarne une condition humaine ambivalente, en porteà-faux, un écart qui est écartèlement.

Celui pour qui la parole est vitale et l'imaginaire un palliatif met toute son énergie créatrice à " transformer un fait divers de deux paragraphes en une tragédie décrivant la scène et la fameuse plage, grain par grain $"{ }^{77}$ La réhabilitation de Moussa passe par l'étayage imaginaire contre une réalité brute perçue comme un affront car incapable de restituer la vérité de Moussa.

Haroun se montrera à la hauteur de l'enjeu qui consiste à « faire vivre Moussa $»^{78}$ puisqu'ayant endossé l'identité de ce dernier, il parvient à produire ce qu'il appelle " un cosmos », c'est-à-dire « la reconstitution imaginaire du crime, la couleur du ciel, les circonstances ... » (131), " une sorte de Mille et Une Nuits du mensonge et de l'infamie » (131), le « livre imaginaire » (131) auquel la mère se raccroche comme à une planche de salut. Un " livre fantôme " ${ }^{79}$ et une identité fantôme qui s'engendrent mutuellement. Issu d'un temps légendaire, le grand hypotexte oriental des Mille et une nuits métaphorise les contes merveilleux ou fantastiques de la mère ${ }^{80}$, composés de "lambeaux de ses rêves ${ }^{81}$; le récit de Haroun ${ }^{82}$ véhicule un message d'espoir puisque l'imaginaire y figure comme protection contre la fatalité. On se souvient de Schéhérazade, l'archétype de la conteuse. Mais « la tombe vide de Moussa » est « un trou " que la mère comble de ses incessantes prières, d'une "fausse biographie ${ }^{83}$ : une sorte de tonneau des Danaïdes. Si elle brode afin de " corriger le réel et [d']atténuer l'absurde ${ }^{84}$, son endurance endolorie et endurcie est sans issue.

Glissements de la parole, polyphonie, affabulation et ellipses : autant de stratégies qui permettent à la contre-enquête de réaliser pleinement sa vocation. En effet, paradoxalement, il faut être fidèle à une "éthique de l'opacité " ${ }^{85}$; Laurent Demanze souligne l'exigence d'opacité dans les récits d'enquête (ou, en l'occurrence, de contre-enquête), où les zones obscures de l'identité ne sont pas clarifiées. S'il y a une " esthétique de la solarité ${ }^{86}$ qui nous ramène à l'univers imaginaire camusien, le traitement en est autre: Haroun fait le choix de l'opacité textuelle contre la solarité impérieuse des protocoles métaphysiques, qu'il rejette autant que les diktats disciplinaires de l'Algérie contemporaine. Créature de l'ombre qui va jusqu'à se confondre avec sa propre ombre ${ }^{87}$ et pour qui Alger elle-même n'est qu'une " ombre ${ }^{88}$, 
Haroun maintient son allégeance au principe dionysiaque (et dionysien), bien qu'il ait été foudroyé par la beauté lumineuse de Meriem. Que la solarité et l'opacité ne soient pas incompatibles, il suffit d'invoquer la figure mythique contradictoire par excellence, Orphée, "divinité de l'ombre et de la lumière, de la vie et la mort, de l'amour et du refus de l'amour ${ }^{89}$ pour s'en assurer.

En fin de compte, l'identité surgit dans tout son paradoxe: plus on la cherche, plus on prend conscience de son caractère labile, comme la mémoire ; on se perd dans un labyrinthe, lieu du détour par excellence, des fausses pistes et de l'errance circulaire. L'œuvre est une galerie des glaces ; Haroun est pris entre deux reflets : Moussa et Meursault.

Le jeu de rôles est un jeu de fantômes avec sa logique de substitution selon laquelle Haroun a remplacé son grand-frère Moussa qui lui-même avait remplacé leur père. ${ }^{90}$ Haroun en vient à parler de " ma résurrection $"{ }^{91}$ Gémellité symbolique qui va encore plus loin puisquelle renvoie à Robinson. Les rôles ne sont pas des pôles opposés puisque Haroun et Meursault sont " les compagnons d'une même cellule dans un huis clos où les corps ne sont que costumes $»^{92}$ : dans l'image de cette incarcération se profile une poignante absurdité qui n'exclut pas non plus le clin d'œil ironique à Sartre. Vertigineux jeu de miroir identitaire puisque Haroun en viendra à s'identifier à ce Meursault pour qui il a une grande aversion. Ironie de toutes les ironies.

La logorrhée taraudée par la douleur renvoie au soliloque de Jean-Baptiste Clamence qui a bien du mal à démêler le vrai du faux. Si Meursault, contre-enquête s'élabore en contrepoint à L'Étranger, la situation d'énonciation tortueusement confessionnelle du roman monologué est empruntée à La Chute qui semble louvoyer entre le speech-act (acte de langage) et la cure psychanalytique ; en fin de compte, il s'agit de la confession ambiguë d'un deuil impossible. On a affaire à une identité paradoxale et à l'ouverture du cadre énonciatif à l'altérité bien que l'assise identitaire soit différente. Le monologue sarcastique est risqué puisque l'autodestruction guette celui qui s'y livre. Haroun s'est engagé dans cette confession interminable pour combler le blanc de toute référence stable mais il court le risque de s'y abîmer. Ce ressassement, qui s'autoproclame divagateur, délirant et, en un ultime défi, « mythomane ${ }^{93}$, constitue la matérialisation imaginaire d'une identité erratique et problématique.

Les lacunes et l'inachèvement nous rappellent que l'indétermination (consubstantielle à l'opacité) est toujours un choix éthique. C'est là aussi que réside la vérité, qui ne peut être que fictionnelle, effilochée et relative. La contre-enquête soulève plus de questions qu'elle n'en résout. L'énigme demeure, dont Meriem serait en quelque sorte l'emblème. Bonne fée émancipatrice rivale d'une mère sorcière castratrice, Meriem aurait pu combler le vide. Réincarnation de la passante baudelairienne, elle représente l'Autre dans cette société où la femme doit nier sa féminité. ${ }^{94}$ Elle fait partie de l'identité fantasmée de Haroun, d'une identité au conditionnel passé. Avec Meriem peut-être aurait-il pu se délivrer...L'intensité de son incandescence laisse entrevoir une identité tangible, positive, une vraie rébellion, mais on connaît le sort qui est réservé à la femme libérée. On ne saura jamais ce qu'il est advenu d'elle. Peut-être même n'est-elle en fin de compte 
qu'un rêve, la créature de rêve aux " cheveux fous » et aux " yeux passionnés » qui viennent « visiter Haroun « dans des rêves insistants ". ${ }^{95}$ Après tout, nous n'en sommes pas à un fantasme ou à un fantôme près.

Tout passe et pourtant « il y a quelque chose qui ne passe pas »: telle est la définition du deuil selon Michel Deguy. ${ }^{96}$ D'où le ressassement. Daoud trouve la solution dans la refiguration, voire la trans-figuration imaginaire, dans une éthique du questionnement (ou un questionnement éthique) qui s'oppose aux réponses toutes faites ou aux identités figées de la victimisation. On aboutit à une identité en question(s), paradoxale, qui sérige contre la doxa. La voix poétique du ressaisissement est celle d'un sujet parlant qui tente d'échapper à la malédiction par la contradiction, seule issue, même si la métamorphose est de nature dialectique.

La littérature se doit de cultiver l'ambiguïté contre les discours simplificateurs, forcément dangereux. Dans le sillage d'Amin Maalouf, Daoud ne cesse de fustiger tout réductionnisme identitaire d'appartenance nationale ${ }^{97}$. Contre l'« identité meurtrière ", Meursault, contre-enquête promeut une conception de l'identité interculturelle, qui sache gérer l'altérité. La notion même de contre-enquête est sous-tendue par le dialogue. Si nous arrivons à quelque éclaircissement que ce soit, celui-ci porte sur la fonction de la littérature, en particulier au regard de l'altérité et de l'alternative qu'elle propose. Daoud s'engage dans une entreprise de démystification de l'ethnocentrisme, de libération de l'imaginaire (celui-ci étant annexé par les intégristes, galvaudé, récupéré à des fins meurtrières). Daoud, c'est Sisyphe attaché à son rocher, voué à l'éternel recommencement de la lutte pour la liberté. La définition de l'écriture romanesque selon Jean-Jacques Wunenburger explicite la métamorphose : "l'activité exemplaire, par laquelle le mythe est arraché à sa sacralité originelle, pour se métamorphoser en œuvre ouverte. [...] La création romanesque, en particulier, se fait à mesure que le mythe de référence se défait ». Wunenburger analyse cette " appropriation " ainsi : « $[\mathrm{t}]$ oute création est d'abord écart, distorsion des formes préexistantes, toute écriture est ré-écriture ${ }^{98}$, avant d'ajouter que la création " contre-dit " ${ }^{99}$ le mythe, ce qui lui confère une valeur de " profanation ». C'est bien la démarche dans laquelle s'inscrit Daoud, soucieux de préserver et de promouvoir l'imaginaire contre tout pervertissement. Profanation qu'il revendique car elle représente le créateur libre et authentique qui s'insurge contre une profanation néfaste et toxique, mortifere et sectaire.

L'imaginaire tourne donc à plein régime : cette contre-enquête nous donne moins à lire une suite à L'Étranger de Camus que l'activation de ses virtualités invisibles et indicibles, des résonances de sa blancheur impassible. Dans l'optique du concept camusien de révolte créatrice, il nous importe de promouvoir le rôle de l'imaginaire dans la quête de la vérité. L'absurde ne se conçoit pas sans la révolte. La contre-enquête est avant tout une quête identitaire, à tendance autobiofictionnelle, poursuivie par Haroun, certes, mais aussi relancée d'un texte à l'autre par Daoud. C'est bien l'imaginaire comme réseau touffu qui permet d'approcher au plus près la vérité. Si l'imaginaire est le point névralgique, pour ainsi dire, de cette herméneutique littéraire c'est parce qu'il 
Imaginaire identitaire/identité imaginaire dans Meursault, contre-enquête de Kamel Daoud

constitue un dépassement de la structure névrotique chez Haroun, une agentivité limitée (mieux assumée, parce que plus aisée chez Daoud, au-delà même de l'universalisme auquel il aspire), un exutoire au trop-plein du deuil dont Moussa n'est que l'objet dicible sur fond d'infini et d'immensité. ${ }^{100}$ Entre les ténèbres de l'intégrisme et $\mathrm{du}$ terrorisme révisionniste et la tyrannie d'un rationalisme tout aussi mortifère que l'absurde dont il est sensé nous préserver, l'imaginaire est dynamisme salutaire: traversée ou dérive, libre circulation qui ouvre à une pensée interculturelle, qui permet de penser l'impensé face au deuil indicible, de dépasser la triade impasse-fantôme-folie. ${ }^{101}$

On n'en finira jamais de se débattre dans ce paradoxe : "Vivre, c'est s'obstiner à achever un souvenir » ${ }^{102}$ Pour l'auteur, comme pour son narrateur (" un vieillard qui ne croit pas en Dieu [...] et qui promène sa liberté comme une provocation » ${ }^{103}$ ), il s'agit de démystifier la doxa par le recours à l'imagination créatrice. C'est en cultivant une vigilance imaginante de tous les instants, bien au-delà de toutes les pirouettes narratologiques et acrobaties oulipiennes, que l'imaginaire, seule solution à l'impasse du modèle victimaire, dangereusement mortifère, offre « un coefficient d'équilibration, de libération et de bonheur ». ${ }^{104}$ Haroun n'invoquait-il pas, en un autre clin d'œil à Camus, « la justice des équilibres " ${ }^{105}$ plutôt que celle des tribunaux? Daoud aura ainsi trouvé le juste milieu entre l'enquête judiciaire et la quête littéraire, dans une contra-diction digne de ce nom...

\section{BibLIOGRAPHIE}

Abraham, Nicolas et Mária Török, L'Écorce et le noyau, Paris, Flammarion, 1987.

Bachi, Salim, Le dernier été d'un jeune homme, Paris, Flammarion, 2013.

Béague, Annick, Jacques Boulogne, Alain Deremetz et Françoise Toulze-Morisset, Les Visages d'Orphée, Paris, Presses universitaires du Septentrion, 1998.

Camus, Albert, L'Étranger, Paris, Gallimard, 1942.

Camus, Albert, La Chute, Paris, Gallimard, 1956.

Char, René, La Parole en archipel, Paris, Gallimard, 1962.

Daoud, Kamel, Meursault, contre-enquête, Actes Sud, 2014.

Daoud, Kamel, « Où en est le rêve algérien ? ", Le Point, publié le 12/01/2020, modifié le 28/01/20 en ligne. bttps://www.lepoint.fr

Daoud, Kamel, «Manuel à l'usage des (re)venants », in Le Point, 2/08/20, modifie 3/08/20. https://www. lepoint.fr

Deguy, Michel, À ce qui n'en finit pas. Thrène, Paris, Seuil, 1995.

Deguy, Michel, «Solutions de continuité ", in Éric Benoit et alii (dir.), Modernités 15. Écritures du ressassement, Bordeaux, PU, 2001.

Djebar, Assia, Le blanc de l'Algérie, Paris, Éditions Albin Michel, 1995.

Guemriche, Salah, Aujourd'hui Meursault est mort : dialogue avec Albert Camus, Éditions Franz Fanon, 2017 (2013 pour l'édition Kindle).

Maalouf, Amin, Les Identités meurtrières, Paris, Grasset, 1998.

Orace, Stéphanie, «L'expression muette du deuil », in Pierre Glaudes et Dominique Rabaté, Modernités 21. Deuil et littérature, Bordeaux, Presses Universitaires, 2005.

Ruffel, Lionel, «Le temps des spectres », in Bruno Blanckeman et Jean-Christophe Millois (dir.), Le roman français aujourd'bui. Transformations, perceptions, mythologies, Paris, Prétexte, 2004. 
Wunenburger, Jean-Jacques, La vie des images, Strasbourg, Presses Universitaires, 1995.

Wunenburger, Jean-Jacques, " Les pères fondateurs de la notion d'imaginaire », in Joël Thomas (dir.), Introduction aux méthodologies de l'imaginaire, Paris, ellipses, 1998.

\section{NoTes}

1. Dorénavant $M C E$.

2. Mot récurrent dans le roman de Daoud. Haroun est taraudé par une question qui ne trouve de réponse que dans l'absurdité : « comment mon frère s'est-il retrouvé sur cette plage ? On ne le saura jamais. Ce détail est un incommensurable mystère et donne le vertige, quand on se demande ensuite comment un homme peut perdre son prénom, puis sa vie, puis son propre cadavre en une seule journée. ", $M C E$, p. 71.

3. «Je voudrais que justice soit faite », affirme d'emblée Haroun, $M C E$, p. 16.

4. Stéphanie Orace, «L'expression muette du deuil», in Pierre Glaudes et Dominique Rabaté, Modernités 21. Deuil et littérature, Bordeaux, PU, 2005, p. 379.

5. Stéphanie Orace, Ibid., p. 376.

6. $M C E$, p. 63.

7. Dans l'édition française du roman de Daoud, Meursault est l'auteur de L'Autre.

8. $M C E$, p. 20.

9. Ibid., p. 74 .

10. $M C E$, p. 130.

11. L'anonymat est décrit par Haroun comme ce «quelque chose qui me sidère », in $M C E$, p. 14 .

12. $M C E$, p. 23.

13. Il se dit uni par le même « destin » : "j'ai moi aussi, une mère et un meurtre sur le dos. ", $M C E$, p. 64.

14. Dès le début (p. 16) et aussi en sa modalité sisyphéenne qui consiste à « pousser un cadavre vers le sommet du mont avant qu'il ne dégringole à nouveau, et cela sans fin. » (p. 57).

15. $M C E$, p. 35 .

16. $M C E$, p. 58.

17. $M C E$, p. 63.

18. Assia Djebar, Le Blanc de l’Algérie, Paris, Éditions Albin Michel, 1995, p. 15.

19. L'image de la mère face à la mer, " comme penchée sur une tombe ", $M C E$, p. 56.

20. $M C E$, p. 49.

21. Qui culmine dans ce que Haroun désigne comme « la profondeur de mon abîme, $M C E$, p. 143.

22. «Ressasser», $M C E$, p. 11 ; « ressassé jusqu'à l'infini », p. 61.

23. La répétition de «cette histoire », en particulier trois fois dans le paragraphe qui ouvre le cinquième chapitre (p. 61) est symptomatique.

24. Nicolas Abraham et Mária Török, L'Écorce et le noyau, Paris, Flammarion, 1987, p. 427.

25. Lionel Ruffel, "Le temps des spectres ", in Bruno Blanckeman et Jean-Christophe Millois (dir.), Le roman français aujourd'hui. Transformations, perceptions, mythologies, Paris, Prétexte, 2004.

26. Dominique Viart. Perec, Modiano

27. $M C E$, p. 63.

28. $M C E$, p. 21.

29. $M C E$, p. 18.

30. $M C E$, p. 150.

31. $M C E$, p. 74.

32. MCE, p. 143. C'est ce jour "maudit» de lété 1942 que Moussa est devenu un «Arabe », p. 71. L'adjectif est omniprésent. Haroun laisse entendre que Moussa était déjà condamné, lui qui aimait sa famille " comme le font les morts, avec un regard venant de l'au-delà », p. 18.

33. $M C E$, p. 46. 
Imaginaire identitaire/identité imaginaire dans Meursault, contre-enquête de Kamel Daoud

34. $M C E$, p. 49.

35. $M C E$, p. 55.

36. $M C E$, p. 11 ; p. 49 ; p. 152.

37. $M C E$, p. 49.

38. $M C E$, p. 144.

39. $M C E$, p. 46.

40. $M C E$, p. 62.

41. Haroun prend soin d'ajouter qu'il s'agit d'un « étrange statut sans sexe, destiné à honorer un deuil éternel », ce qui fait d'elle une veuve hyperbolique, « épouse de la mort » plus que d'un mort, p. 49.

42. $M C E$, p. 48.

43. $M C E$, p. 31.

44. $M C E$, p. 59.

45. $M C E$, p. 70.

46. $M C E$, p. 72.

47. $M C E$, p. 75 .

48. MCE, p. 133.

49. $M C E$, p. 67.

50. $M C E$, p. 69. Ajoutons que Haroun redoute la mer qui le « dévore trop vite » (p. 65) et abhorre Alger dont la baie est métaphorisée en une mâchoire, et « son monstrueux bruit de mastication », $M C E$, p. 149.

51. $M C E$, p. 97.

52. MCE, p. 98.

53. $M C E$, p. 98.

54. Kamel Daoud, « Où en est le rêve algérien ? », Le Point, publié le 12/01/2020, modifié le 28/01/20 en ligne.

55. MCE, p. 108.

56. Pour reprendre le titre du tombeau poétique de Michel Deguy, À ce qui n'en finit pas. Thrène, Paris, Seuil, 1995.

57. MCE, p. 131.

58. MCE, p. 131.

59. $M C E$, p. 56.

60. $M C E$, p. 57-8.

61. $M C E$, p. 51.

62. MCE, p. 131.

63. $M C E$, p. 21.

64. Kamel Daoud, « Manuel à l'usage des (re)venants », in Le Point, 2/08/20, modifie 3/08/20.

65. $M C E$, p. 50.

66. $M C E$, p. 52.

67. $M C E$, p. 77.

68. $M C E$, p. 132-3.

69. $M C E$, p. 134.

70. $M C E$, p. 77.

71. $M C E$, p. 138.

72. MCE, p. 139.

73. MCE, p. 138.

74. Elle est liée au divin, $M C E$, p. 150.

75. MCE, p. 134

76. MCE, p. 134.

77. MCE, p. 130.

78. MCE, p. 131. 
79. $M C E$, p. 132.

80. $M C E$, p. 26.

81. $M C E$, p. 52.

82. $M C E$, p. 131.

83. $M C E$, p. 57.

84. $M C E$, p. 46-7.

85. Laurent Demanze, Un nouvel âge de l'enquête. Portraits de l'écrivain contemporain en enquêteur, Paris, José Corti, p. 277.

86. Ibid., p. 261.

87. $M C E$, p. 66.

88. $M C E$, p. 62.

89. Annick Béague et alii, Les Visages d'Orphée, Paris, Presses universitaires du Septentrion, 1998, p. 27.

90. $M C E$, p. 20.

91. $M C E$, p. 100.

92. $M C E$, p. 20.

93. MCE, p. 153.

94. Zoubida (dont Haroun nous apprend qu'elle est sans doute liée à Moussa) est cette « femme mystérieuse » (p. 30) qui, sitôt entrevue, disparaît (p. 31) : une apparition qui mérite certes une attention plus soutenue que celle que nous lui portons.

95. $M C E$, p. 77.

96. "Solutions de continuité », in Éric Benoit et alii, (dir.), Modernités 15. Écritures du ressassement, Bordeaux, PU, 2001, p. 91.

97. Amin Maalouf dénonce une conception « qui réduit l'identité à une seule appartenance, installe les hommes dans une attitude partiale, sectaire, intolérante, dominatrice, quelques fois suicidaire ", in Les Identités meurtrières, Paris, Grasset, 1998, p. 39.

98. Jean-Jacques Wunenburger, La vie des images, Strasbourg, PU de Strasbourg, 1995, p. 54.

99. Ibid., p. 54.

100. Haroun fait de l'infini un « contrepoint » qui sert à « équilibrer notre condition humaine », $M C E$, p. 59.

101. La reconstitution du crime sur la plage aboutissait à « une impasse, à un fantôme, à la folie », $M C E$, p. 67.

102. René Char, La Parole en archipel, Paris, Gallimard, 1962, p. 84.

103. $M C E$, p. 150.

104. Jean-Jacques Wunenburger, "Les pères fondateurs de la notion d'imaginaire ", in Joël Thomas (dir.), Introduction aux méthodologies de l'imaginaire, Paris, ellipses, 1998, p. 119.

105. MCE, p. 16. 